\title{
An Interprofessional Education Pilot Program on Screening, Brief Intervention, and Referral to Treatment (SBIRT) Improves Student Knowledge, Skills, and Attitudes
}

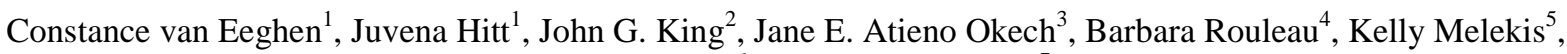 \\ Rodger Kessler ${ }^{6} \&$ Richard Pinckney ${ }^{7}$ \\ ${ }^{1}$ General Internal Medicine-Research, Robert M. Larner MD College of Medicine, The University of Vermont, 89 \\ Beaumont Avenue S456, Burlington, VT, USA \\ ${ }^{2}$ Department of Family Medicine, Robert M. Larner MD College of Medicine, The University of Vermont, 89 \\ Beaumont Avenue S456, Burlington, VT, USA \\ ${ }^{3}$ Department of Leadership and Developmental Sciences, College of Education \& Social Services, The University of \\ Vermont, Mann Hall 101B, Burlington, VT, USA \\ ${ }^{4}$ Department of Nursing, College of Nursing \& Health Sciences, The University of Vermont, Burlington, VT, USA \\ ${ }^{5}$ Social Work Department, Skidmore College, 815 N. Broadway, Saratoga Springs, NY, USA \\ ${ }^{6}$ Behavioral Health Program, College of Healthcare Solutions, Arizona State University, Phoenix, AZ, USA \\ ${ }^{7}$ Department of Internal Medicine, Robert M. Larner MD College of Medicine, The University of Vermont, 89 \\ Beaumont Avenue S456, Burlington, VT, USA \\ Correspondence: Constance van Eeghen, General Internal Medicine Research, Robert M. Larner MD College of \\ Medicine, The University of Vermont, 89 Beaumont Avenue S456, Burlington, VT 05405, USA. E-mail: \\ cvaneegh@med.uvm.edu
}

Received: January 16, 2019

Accepted: February 12, 2019

Online Published: February 14, 2019

doi:10.5430/ijhe.v8n1p119

URL: https://doi.org/10.5430/ijhe.v8n1p119

\begin{abstract}
Background

Recent efforts to prepare healthcare professionals to care for patients/clients with substance use problems have incorporated SBIRT (Screening, Brief Intervention, and Referral to Treatment) into graduate education programs. This pilot study adds to the literature by examining the impact of an SBIRT interprofessional education approach for behavioral health graduate students and medical residents as planned by faculty from multiple professions at a state university. It measured changes in attitudes, abilities, skills, and knowledge in these interprofessionally trained students.
\end{abstract}

\section{Methods}

Faculty in Counseling, Family Medicine, Internal Medicine, Nursing and Social Work departments collaborated to develop an interprofessional curriculum delivered through a small-group and active learning approach. Seventy-one residents and graduate students participated. Pre- and post-training surveys (Note 1) measured self-perceived attitudes, abilities, and skills along with objectively measured knowledge. Analysis examined pre- to post-training changes in scores.

\section{Results}

Pre-training surveys yielded an $89 \%$ response rate; post-training, $85 \%$. Self-perceived attitudes did not change significantly, except a $20 \%$ increase in how rewarded students felt while working with patients/clients with alcohol/drug use disorders $(\mathrm{P}<.01)$. Compared to baseline, there was a statistically significant increase in all items of self-perceived ability $(\mathrm{P}<.01)$ and all items of self-perceived communication skills $(\mathrm{P}=.04)$. Knowledge mean scores also increased significantly $(\mathrm{P}<.001)$ across both primary care and behavioral health student groups. 


\section{Conclusions}

Interprofessional training in SBIRT produced improvements in ability, skills, knowledge, and some attitudes. Such programs may inform providers who care for patients/clients with substance use problems, improving their personal experience and professional competence.

Keywords: motivational interviewing; substance-related disorders; interprofessional education; counseling education; medical education; nursing education; social work education; curriculum

\section{Introduction}

Alcohol and drug use disorders affect morbidity and mortality across the globe. Annually, 3.3 million deaths worldwide are attributed to alcohol use disorder (WHO, 2015). In the U.S., drug-related overdoses increased by $540 \%$ between 1980 and 2010. The number of drug overdose deaths per year increased 23\%, from 38329 in 2010 to 47 055 in 2014 (Warner, Trinidad, Bastian, Minino, \& Hedegaard, 2016). Screening, Brief Intervention, and Referral to Treatment (SBIRT) is a "comprehensive, integrated, public health approach to the delivery of early intervention and treatment services for adolescents and adults dealing with substance misuse (including use disorder) issues" (Babor et al., 2007, p. 8). The SBIRT program serves the targeted substance users who are not yet dependent and could reduce drug use through early intervention (Gryczynski et al., 2011; Madras et al., 2009). The SBIRT program has been shown to reduce alcohol consumption and consequences of abuse (APHA, 2008).

\subsection{Role of Interprofessional Education (IPE)}

Health care professionals will need to provide SBIRT interventions in interdisciplinary settings (Agerwala \& McCance-Katz, 2012; Davoudi \& Rawson, 2010) and on teams (Cleak \& Williamson, 2007; Newhouse \& Spring, 2010). Interprofessional education (IPE) can enhance SBIRT programs and benefit all learners training to work in such contexts substantially. The Health and Medicine Division of the National Academies supports IPE-enhanced training to improve patient/client outcomes through better utilization of the shrinking health care workforce (Corrigan, 2005). Though graduate medical education programs have successfully trained residents in SBIRT, most of the studied programs are not interprofessional. (Note 2)

\subsection{IPE Literature}

The terms "interdisciplinary" and "interprofessional" are often used interchangeably in the literature, but there are substantial differences. "Interdisciplinary" has been defined as a branch of knowledge or field of study. Multiple disciplines can be found within a single profession such as medical doctors with specialty disciplines in medicine, pediatrics, psychiatry, dentistry, etc. (Bray et al., 2012; Neufeld et al., 2012; Pringle et al., 2012; Tetrault et al., 2012). The term "interprofessional" relates to a vocation or profession requiring specialized knowledge and skills, such as medicine, nursing, counseling and social work (IEC, 2011). Olenick, Allen, and Smego (Olenick, Allen, \& Smego, 2010) report that interdisciplinary behavior lacks the depth of collaboration implied by the term "interprofessional."

The World Health Organization (Gilbert, Yan, \& Hoffman, 2010) defines IPE as "when students from two or more professions learn about, from, and with each other to enable effective collaboration and improve health outcomes" ( $p$. 196). Interprofessional education is increasingly included as a required component in university accreditation standards for health professions in order to prepare a "collaborative practice-ready health workforce" (Gilbert et al., 2010 , p. 196). A collaborative practice-ready health worker is someone who has learned how to work in an interprofessional team and is competent to do so. The Interprofessional Collaborative Expert Panel (2011) cited four core competencies for Interprofessional Collaborative Practice. The four competency domains are: 1) Values and Ethics; 2) Roles and Responsibilities; 3) Interprofessional Communication; 4) Teams and Teamwork.

Because collaboration between professions is critical to improving quality, safety, and access to care, providing opportunities for interprofessional learning experiences can help prepare future professionals for a team approach to providing care (Hackett, 2015). A growing number of studies suggest that IPE improves students' confidence in their professional role (Mann, 2009), attitudes toward and knowledge about other professions and toward IPE (Bolesta \& Chmil, 2014; Pelling, Kalen, Hammar, \& Wahlstrom, 2011), and team and clinical reasoning skills (Robben et al., 2012; Seif et al., 2014). Students who interact with other health professions learn to value diverse perspectives, respect the knowledge and expertise of other professions, collaborate when problem-solving, and communicate as a team to ensure patient safety (Olenick et al., 2010; Solomon \& Salfi, 2011).

As a result of the need for IPE, a number of investigative educators have developed and studied interprofessional training for students to better prepare them for the workforce (Duong, O'Sullivan, Satre, Soskin, \& Satterfield, 2016; A. M. Mitchell et al., 2018; Monteiro et al., 2017; Neander et al., 2018; Neft, 2018; Peterson, 2017). Collectively, 
these programs have demonstrated that interprofessional trainings have high satisfaction among students and enhance interprofessional competence, condition specific knowledge, skill, and attitudes around SBIRT and working with patients with substance use disorders. However, these interprofessional SBIRT programs have differed extensively in the degree to which the students interacted, how much the students represent the true composition of professionals doing this work, and the degree to which faculty role modeled interprofessional collaboration.

When reviewing the research to date, a pattern emerges of a possibly optimal intervention: synchronous, face-to-face training, in which interprofessional students from all appropriate disciplines work together, using standardized patients that represent complex real-world scenarios (e.g. patients have interacting substance use disorders (SUD) and chronic medical conditions), and fully interprofessional faculty who role-model collaboration as part of instruction. There is one study that possesses all of these attributes. Peterson, Brommelsiek, and Amelung (Peterson, 2017) trained a cohort of learners which included students of advanced practice nursing, pharmacy, clinical psychology, and social work. They found that students in this program valued the team approach and experienced high levels of communication, cooperation, and collaboration during the training. What is unknown is whether this training enhanced student attitudes, skills, or knowledge. An interactive IPE curriculum showing measurable results in supporting a collaborative workforce that is knowledgeable, skilled, and confident in its practice of SBIRT would help fill this gap in the field.

\subsection{Purpose of Study}

The University of Vermont (UVM) SBIRT Training Collaborative piloted an interprofessional curriculum that builds on this work by training a similar group of students (counseling, medical residents, nursing, and social work) using a similar approach and evaluating the impact of this training on student attitudes, abilities, skills, and knowledge. Faculty who developed and delivered the program represented all of the four professions. The program began with a brief didactic session at program level, followed by two 2-hour workshops which included an interprofessional faculty panel, standardized patients, and small interprofessional group work. The faculty panel provided role modeling and discussion of various contexts for SBIRT. The standardized patients engaged students in practicing brief negotiated interviews and the small groups allowed students to learn from each other about referring patients/clients and working as a team. The theme of learning from other professions and the importance of clinical context for applying SBIRT permeated all elements of the training. We further advanced this field by observing differences among medical and behavioral health service students in their response to this training. This work was supported by the Substance Abuse and Mental Health Service Agency (SAMHSA) 2014-2017.

\section{Methods}

\subsection{Procedures}

To pilot this work, the interprofessional SBIRT Advisory Council oversaw the development of the interprofessional education SBIRT curriculum, beginning in 2013. This council included representation from five departments (Counseling, Family Medicine, Internal Medicine, Nursing, and Social Work) in three UVM colleges (Education and Social Services, Nursing and Health Sciences, and Medicine). The Council endorsed the four domains of IPE core competencies (Table 1) and imbedded the curricula into each discipline except Social Work, in which participation in the SBIRT program was voluntary. Using a flipped-classroom model, most students completed an online didactic training which was then followed by a series of two 2-hour interprofessional workshops. The workshops occurred approximately 1 month apart. Using videos and role-playing exercises with standardized patients, students learned screening for risky substance use, communication styles and strategies for brief intervention based on a brief motivational interviewing approach, and referral collaboration methods. Training in brief negotiated interviews followed recommendations of the Motivational Interviewing Network of Trainers (MINT) (2016). A MINT faculty member served on the SBIRT Advisory Council and contributed to the development of the training program. A faculty development workshop preceded the training at which faculty from all departments were engaged in a review of the workshop content and teaching methods.

\subsection{Training site and Students}

The University of Vermont's Simulation Laboratory provided real time, practical experience to support SBIRT and team-building skills. The nine-month training program engaged first-year residents and first- or second-year graduate students in counseling, nursing, and social work (Table 2). The Committees on Human Research at UVM reviewed the study protocol and determined that that it did not require IRB review under 45 CFR 46.102(d). 
Table 1. Interprofessional Education Domains and Core Competencies included in training program

\begin{aligned} \hline Values/Ethics & \\ & $\diamond$ Patient-centered care planning \\ & $\diamond$ Mutual respect for other professions \\ Roles/Responsibilities & $\diamond$ Recognize one's limitations in skills, knowledge and abilities \\ & $\diamond$ Engage diverse professionals to complement one's own expertise \\ & $\diamond$ Use unique and complementary abilities of all members of the team \\ & $\diamond$ Confident expression of one's knowledge and opinions \\ & $\diamond$ Listen actively \\ & $\diamond$ Encourage ideas and opinions of others \\ & $\diamond$ Give timely, sensitive, instructive feedback \\ Tnterprofessional Communication & $\diamond$ Engage and integrate other professionals in patient-centered care \\ & $\diamond$ Share accountability \\ Teams/Teamwork & \end{aligned}

As shown in Table 1, four interprofessional competency domains as published by The Interprofessional Education Collaborative (2011) can guide training initiatives.

Table 2. Professions and demographic characteristics of the 67 SBIRT student respondents at the University of Vermont, (Spring 2015)

\begin{tabular}{lc}
\hline Profession & $\mathrm{N}$ \\
\hline Total & $67^{*}$ \\
Counseling $(\%)$ & $12(18)$ \\
Family Medicine & $4(6)$ \\
Internal Medicine & $12(18)$ \\
Social Work & $17(25)$ \\
Nursing & $22(33)$ \\
\hline Demographics $(\%) * *$ & \\
\hline Female & $49(73)$ \\
Hispanic/Latino & $3(4)$ \\
Black/African American & $1(1)$ \\
Asian & $3(4)$ \\
White & $57(85)$ \\
American Indian & $1(1)$ \\
Not Reported & $7(10)$
\end{tabular}

* 4 students did not complete either a pre-training or a post-training survey

** Total sums for race total $>67$ as respondents chose all that applied

As shown in Table 2, students who completed surveys both before and after the training program represented all professions involved in this project, as well as an array of demographic characteristics. 


\subsection{Didactic Education Program}

Students from each department received uniform didactic education using the SAMHSA SBIRT curriculum. Didactic curriculum (85 minutes plus optional videos) provided by SAMSHA via an Oregon Health and Science University website (http://www.sbirtoregon.org), or through in-person interprofessional instruction (2 hours) developed at our institution, provided basic information on alcohol and drug use, prevalence, morbidity and interventions, and screening workflows/tools for adolescents and adults. Those receiving in-person interprofessional instruction were exposed to the same content as other students; however, it was delivered in a two-hour, small group session which featured didactics, a demo, and discussion. This session was taught by a member of MINT.

\subsection{Interprofessional Session 1}

The first in-person IPE session provided a faculty panel discussion, motivational interviewing (MI) demonstration, team-based training with standardized patients, and debriefing with faculty and program staff. The goal of this 2-hour session was to increase student skills in applying SBIRT to clinical scenarios. Objectives included:

1. Understand the different clinical contexts in which SBIRT can be used

2. Interpret the results of screening tools

3. Conduct a brief negotiated interview

4. Examine the role of diversity in the interview and intervention process

5. Explore each other's professional roles and responsibilities through IPE

Faculty panel discussions presented varying contexts in which disciplines may screen for substance use and utilize MI skills. A representative from each profession discussed 1) their training in SUD, 2) work settings and types of routine screening/assessments appropriate for SBIRT, 3) scenarios that prompt screening for SUD, 4) common barriers to SBIRT, and 5) situations particularly assisted by interprofessional collaboration. Following the panel, students observed a demonstration of a brief negotiated interview and asked questions about MI skills and strategies.

Faculty-facilitated interprofessional groups of 5-8 students, randomly assigned, engaged in active learning with trained standardized patients (SPs) enacting three substance misuse scenarios in 15-minute segments. Students prepared by reviewing handouts with a patient/client profile, case description, and results of pre-screening tools for alcohol and drug misuse (Gache et al., 2005; Yudko, Lozhkina, \& Fouts). In small groups, students collaborated to gather relevant history including detailed substance misuse screening. They practiced brief negotiated interviews as indicated by the case. Following each interview, a faculty member debriefed students and SPs, reviewing interview strengths and alternative strategies as well as encouraging discussion of approaches used by different professions. Facilitators helped students consider all aspects of the case (medical, psychosocial, etc.), work as an interprofessional team, respect each other's professions and scope of practice, and demonstrate awareness and respect for cultural/identity differences for each patient/client.

\subsection{Interprofessional Session 2}

The second 2-hour interprofessional session focused on "referral to treatment" through a faculty panel discussion, review of cases from session one, and debriefing. The goal was to provide insight to the individualized needs of patients/clients regarding substance misuse and approaches to meet those needs through an interprofessional team. Objectives included:

1. Demonstrate an understanding of the roles of various professions in the management of SUD

2. Explain the reasons for initiating a referral for patients/clients in each setting

3. Consider a range of factors in making referrals to other professionals

During panel discussion, faculty discussed contextual and discipline-specific factors in making referrals. A representative from each profession discussed 1) the referral process in relation to their profession, 2) common hurdles, 3) strategies for a successful referral, e.g. "warm handoff," and 4) approaches to a comprehensive care plan including treatment from other providers/specialists. Materials included guidelines for referral-to-treatment and resources for additional training. Students joined interprofessional active learning groups of 4-6 students to revisit the cases from session one, focusing on strategies for advancing the initial intervention. (Note that group sizes were adjusted between sessions to accommodate the number of faculty volunteers and students present.) Using these strategies, students planned referrals and evaluated their benefits in addressing SUD. The faculty facilitators were encouraged to explore the role of different professionals in intervening with SUD cases and how they can work 
together as a team to address patient needs. During debrief, faculty and students addressed questions about the process of referral and how to continue to use and develop SBIRT skills.

\subsection{Survey Methods}

We measured student satisfaction with each session using SAMSHA's GPRA survey (SAMHSA, 2017) immediately following the session. Pre/post surveys assessed changes in attitudes, abilities, skills, and knowledge of working with patients/clients with drug or alcohol misuse. Students also accessed pre-training surveys through REDCap, a secure, web-based application hosted at UVM. (Note 3) Post-training, students received paper surveys at their training sites.

\subsection{Questionnaire Development}

We constructed a pre and post survey that measured attitudes, abilities, and skills based on previous work of SBIRT training programs at the University of Pittsburgh (Venkat et al., 2017). From the original items and domains, we selected those representing the domains of attitudes, abilities, and skills. All assessment items used a 5-point Likert scale. "Attitudes" included items that reflected an underlying belief system about working with patients/clients with use disorders. "Abilities" reflected overall perception of knowledge, skill, and experience. "Skills" isolated the communications skills, e.g. to raise the topic of quantity/frequency of alcohol or other drug use and ask the patient to self-assess.

In addition, we developed a 32-item test of SBIRT facts for pre/post comparisons of students' knowledge. The test presented questions in "multiple choice single best answer" format or "check all that apply." The latter were analyzed dichotomously, as individual items for each possible response. The test contained questions based on didactic content, including the effects of drugs/alcohol, screening strategies, and brief negotiated interventions. The complete survey of "attitudes, abilities, and skills" and "student knowledge" is available as Supplemental Digital Appendix 1. The survey was administered twice (pre/post) in the second year of the program, from 2014-2015.

\subsection{Analysis}

We analyzed the Likert scale items for attitudes, abilities, and skills by subtracting pre-training from post-training scores and conducting one-sample testing with a comparison to a null of 0 , or no difference. We used a t-test when assumptions of normality were met and the sign-rank test as a non-parametric alternative. To summarize our findings, we dichotomized the Likert scale by presenting the proportion of participants agreeing with competence and attitudinal statements (scores of 4 or 5).

Similarly, we analyzed knowledge scores by comparing differences between pre- and post-training scores, comparing to a null of 0 , and assessing for statistical significance using either one-sample t-test or sign rank tests as appropriate. We conducted subgroup analysis by grouping students into behavioral health $(\mathrm{BH})$ practitioners (counseling and social work) and primary care (PC) practitioners (family practice, internal medicine and nursing). All testing was two-tailed with $\propto=.05$, using STATA 12.0 (StataCorp, Stata. 2011, StataCorp LP: College Station, TX).

\section{Results}

\subsection{Students}

A total of 71 students completed the training in the course of an academic year, each assigned to one of three IPE sections, with 67 completing one or both surveys. Sixty-three students (28 BH and $35 \mathrm{PC}$ ) completed the pre-training survey ( $89 \%$ response rate) and sixty (25 BH and $35 \mathrm{PC}$ ) completed the post-training survey (85\%). Fifty-five students completed both sets of evaluation and are included in the analyses. Student satisfaction was high. After session one, $89.6 \%$ of students were satisfied with the overall quality and $86.6 \%$ would recommend the training to a colleague. Students were similarly satisfied with session two; $88.1 \%$ were satisfied with the overall quality of and $86.6 \%$ would recommend the training to a colleague. There were no significant differences between behavioral health and primary care trainees in these satisfaction ratings.

\subsection{Self-Perceived Attitudes}

Most self-perceived attitudes did not change significantly with the exception of two items showing a significant increase in students' perceptions that working with patients/clients with alcohol or SUD was rewarding. Before training, $41 \%$ of students agreed that working with patients/clients with alcohol use disorder was rewarding; afterwards, $61 \%$ agreed, $\mathrm{P}=.04$. The findings for substance use disorder were similar (Figure 1). Both PC students and $\mathrm{BH}$ students experienced this boost. However, the two student subgroups were significantly different from each other, before and after training. Pre-training, 54\% of $\mathrm{BH}$ students agreed that working with those with alcohol use 
disorder was rewarding while only $30 \%$ of PC clinicians agreed, $\mathrm{P}=.04$. Post-training, agreement increased to $79 \%$ in the BH students and $47 \%$ in the PC students, $\mathrm{P}<.01$. Changes seen in drug use disorders were similar, with an increase from $35 \%$ to $59 \%$ in students who found working with drug use disorders rewarding, $\mathrm{P}<.01$. At baseline the two student groups differed, with $50 \%$ of $\mathrm{BH}$ students and $23 \%$ of PC clinicians finding this rewarding, $\mathrm{P}<.01$. Post-training, $79 \%$ of BH students and $43 \%$ of PC clinicians found working with drug use disorders rewarding, P $<.01$.

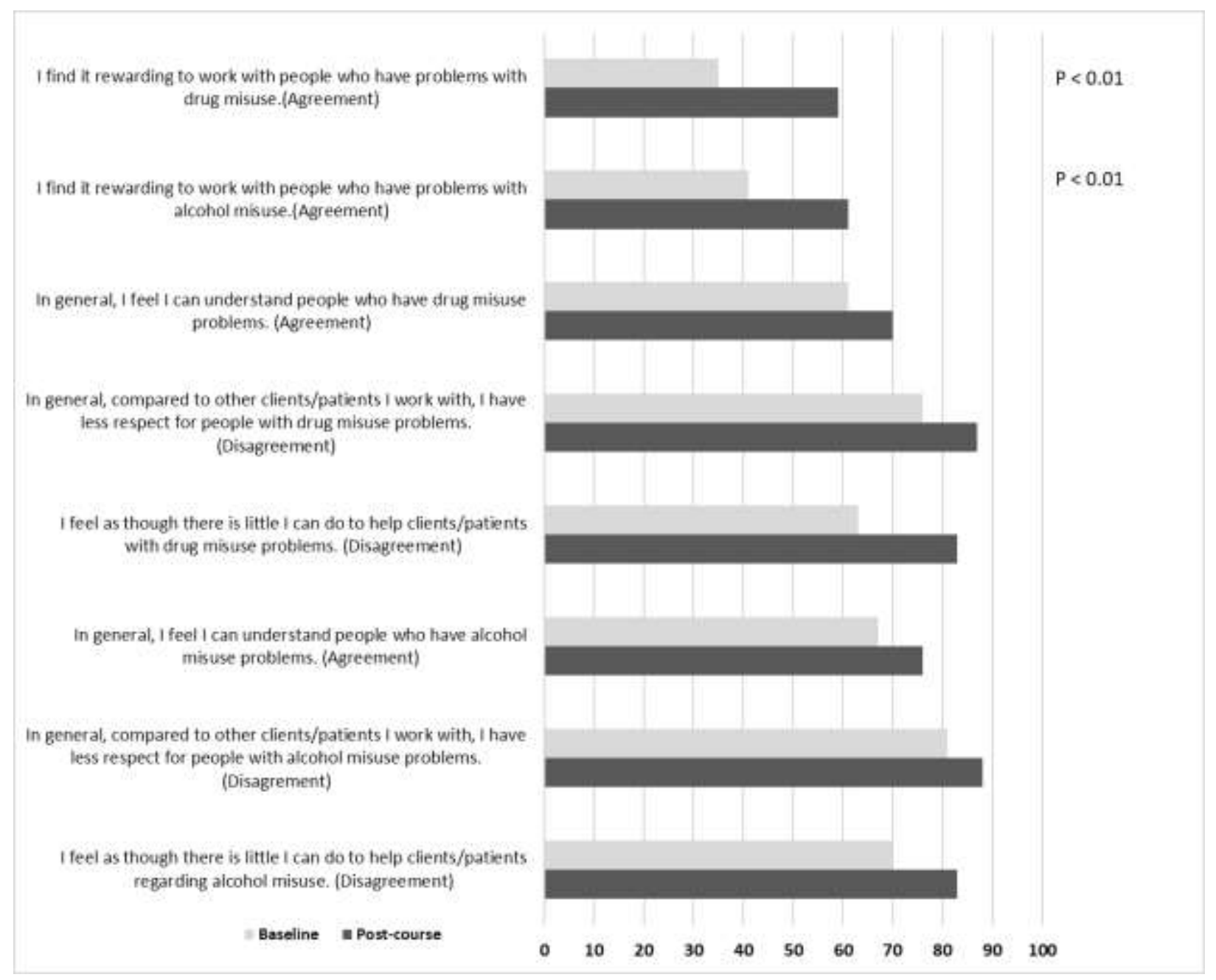

Figure 1. Changes in Self-Perceived Attitudes

Legend: Compares pre-course attitudes of 55 students at the University of Vermont to post-course average scores in Spring 2015. P values indicated only when less than or equal to 0.05 .

\subsection{Self-Perceived Ability (Knowledge, Skills, Experience)}

All measures of self-perceived ability increased after training (Figure 2). For example, only $44 \%$ of students felt they could appropriately advise patients/clients about the effects of drugs prior to the workshop, which increased to $70 \%$ after the workshop, $\mathrm{P}<.01$. At baseline, the lowest rated ability (working with people with drug misuse) found $19 \%$ of students satisfied. Post-training, this increased to $52 \%, \mathrm{P}<.01$. Interestingly, these baseline and post-workshop rating changes were exactly equal in $\mathrm{BH}$ and $\mathrm{PC}$ clinicians. 
On the whole, I am satisfied with the way I work with people who have problems with drug misuse.(Agreement)

I feel I can appropriately advise my clients/patients about the effects of drug misuse.(Agreement)

I feel as though I have a working knowledge or problems related to drug misuse.(Agreement)

On the whole, I am satisfied with the way I work with people who have problems with alcohol misuse.(Agreement)

I feel I can appropriately advise my clients/patients about the effects of alcohol misuse.(Agreement)

I feel as though I have a working knowledge of problems related to alcohol misuse. (Agreement)

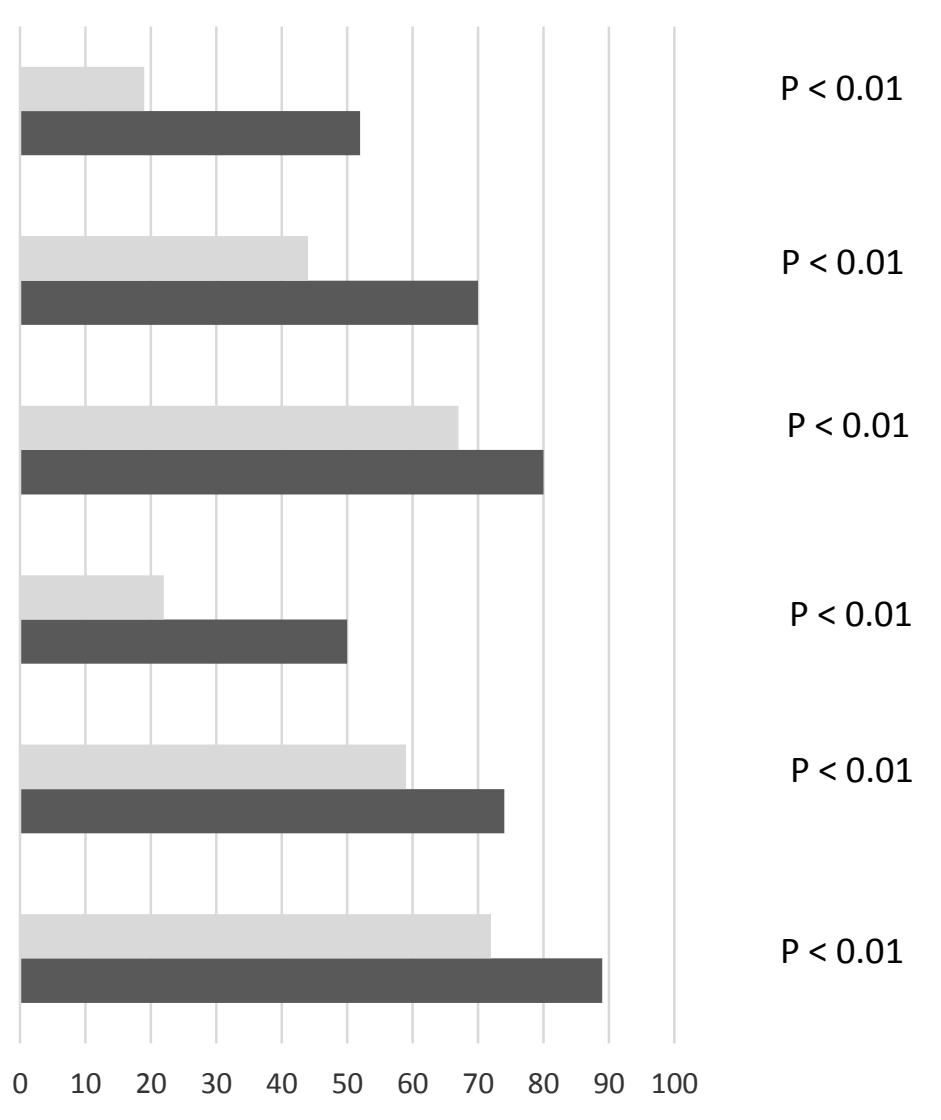

Baseline $\square$ Post-course

Figure 2. Changes in Self-Perceived Ability

Legend: Compares pre-course ability of 55 students at the University of Vermont to post-course average scores in Spring 2015. P values indicated only when less than or equal to 0.05 .

\subsection{Self-Perceived Communication Skills}

We found statistically significant improvements in all five measures of self-perceived communication skills competence (Figure 3), with at least $80 \%$ of students feeling competent post-training. The most striking improvements in feeling competent included asking about behavioral health change (rising from $35 \%$ before training to $85 \%$ after, $\mathrm{P}<.01$ ) and asking about client/patient need to change their use (increasing from $54 \%$ to $80 \%, \mathrm{P}$ $=.002$ ). 
Discuss change with clients/patients regarding their alcohol or drug use behavior as determined by patient/client need.

Ask clients/patients if they think that there is a need to change their alcohol and drug use as determined by patient/client need.

Ask clients/patients if they see their substance use as a problem as determined by patient/client need.

Asking about quantity and frequency of alcohol or other drug use.

Ask clients/patients about alcohol and drug use.

Baseline Post-course

0

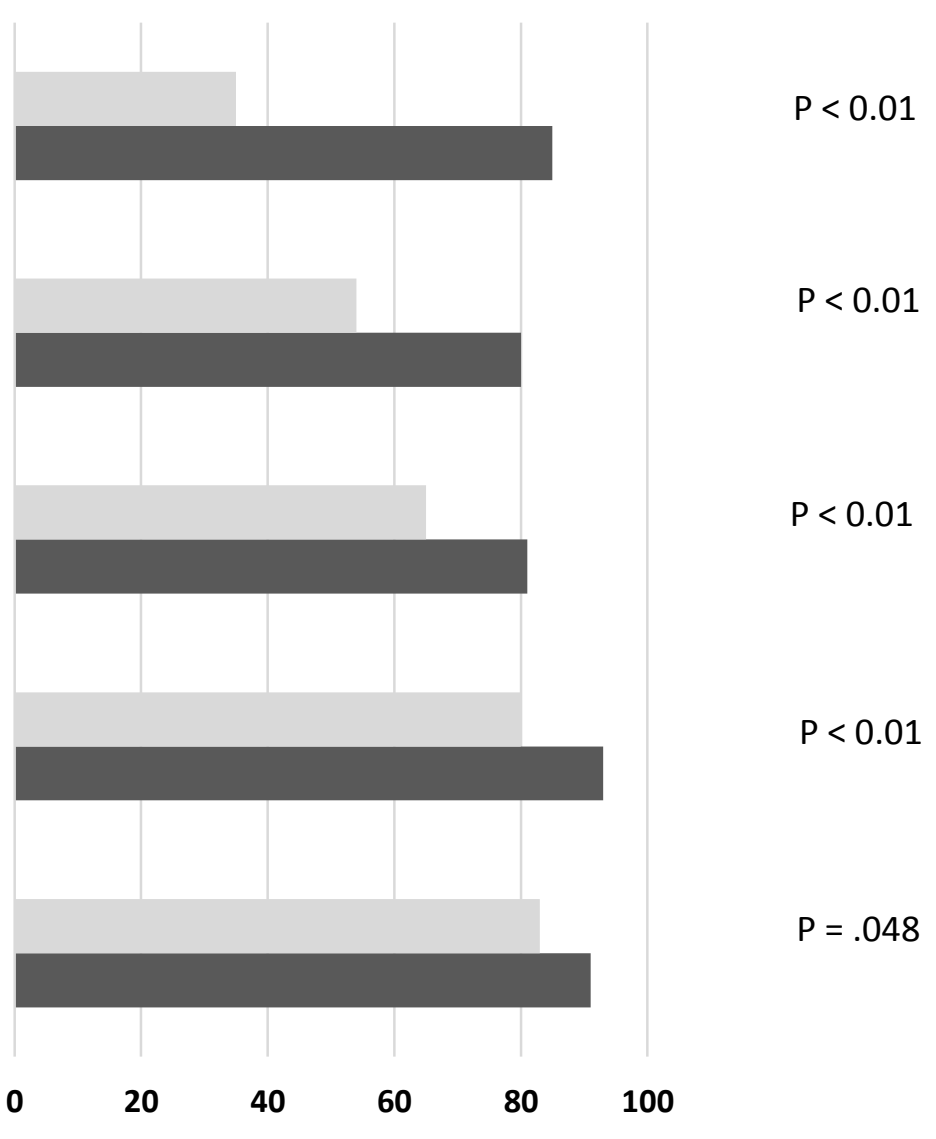

Figure 3. Changes in Self-Perceived Communication Skills Competence

Legend: Compares pre-course communication skills of 55 students at the University of Vermont to post-course average scores in Spring 2015. P values indicated only when less than or equal to 0.05 .

Differences between student types appeared only regarding competence in "asking clients about alcohol and drug use." Pre-training, $75 \%$ of $\mathrm{BH}$ students felt competent compared to $90 \%$ of PC students, $\mathrm{P}=.01$. BH students responded more strongly to training, $\mathrm{P}=.01$, resulting in no significant difference between the two groups post-training.

\subsection{Test Score Knowledge}

Based on test scores, students' knowledge changed significantly. The "knowledge" mean score increased from 27 to 28 points out of a possible 32, (interquartile range for change $0-3) \mathrm{P}<.001$. Primary Care students had slightly higher knowledge pre-training scores (mean $(\mathrm{SD})=28.1(1.6)$ ) than those for $\mathrm{BH}$ students $(25.8(2.7)$ ). There was not a statistically significant difference between $\mathrm{PC}$ and $\mathrm{BH}$ students on the change in knowledge but the two groups remained significantly different in their final scores with an average of 28.9 points for PC providers and 27 for BH providers, $\mathrm{P}=.008$. The standardized mean difference in scores was 0.53 (Lakens, 2013).

\section{Discussion}

A comprehensive IPE-based SBIRT curriculum was feasible and produced a successful approach to educating health care professionals. The curriculum included a self-paced introductory session delivered primarily online prior to face-to-face work groups. Two 2-hour interprofessional sessions with expert faculty provided students with practice opportunities and discussions focused on SBIRT methodology and individualized patient/client treatment planning. This curriculum allowed faculty and students across multiple professions to work together and learn from each other. The findings of this study reinforced literature recommendations that effectively addressing substance use in clinical practice calls for interprofessional teams and settings (Agerwala \& McCance-Katz, 2012; Cleak \& Williamson, 2007; Davoudi \& Rawson, 2010; Newhouse \& Spring, 2010). The success of this pilot resulted in a 
formal decision by the various departments at the University of Vermont to continue the program beyond the funding of the grant; the program is currently in its fifth year.

One interesting finding is that while knowledge, abilities, and skills seemed to improve, attitudes were less influenced by the training. Only two of the attitudinal measures showed improvement as a result of the training. One explanation is that attitudes may be harder to change in response to a training of this type. Also, some of the baseline items related to attitude were already high at baseline. Six of the eight attitudes measures had $60 \%$ or higher agreement at baseline, making it harder to improve.

Some interesting differences emerged between behavioral health students (social work and counseling) compared to the primary care students (nursing students and residents). The primary care providers had higher baseline and post workshop scores on knowledge. This is likely because this represents a post-graduate primary care audience with several years of clinical experience compared to the mostly first-year behavioral health masters students. A similar pattern was seen at baseline in feeling competent to ask clients about alcohol and drug use. The behavioral health providers felt less competent than the primary care providers. This may be due to the regular inclusion of alcohol and drug use assessment in the $\mathrm{PC}$ role, whereas this responsibility is not a usual function of a $\mathrm{BH}$ practitioner unless specializing in substance use treatment. In this case, the training actually improved the behavioral health provider's competence so much that there was no significant difference between the two groups by the end of the training. What was also reassuring to see is that both groups expressed equally high satisfaction with the training.

Our program adds to the growing evidence that education about SBIRT can and perhaps should be offered in an IPE curriculum that fosters team-based care. Building on previous studies, we demonstrated that bringing an interprofessional cohort of students together with an interprofessional, collaborative team of faculty produced significant changes in outcome as measured by attitude, ability, skills, and knowledge. The approach should enhance the ability of teams trained through this method to provide effective substance use screening and interventions. Furthermore, this team-based program can embrace medical conditions as they relate to behavioral and SU conditions.

\subsection{Limitations}

Limitations include an absence of a control group to measure secular trends. This was the only known SUD education for these students during this period; we presumed that changes seen were the direct result of training. Trainees may have had varied education about or experience with SUD beforehand or concurrently, affecting differences in outcomes. Perceptual measures were based on self-report, not observation, with no follow-up assessment of skills and attitudes to reflect retention. The data represent one trainee cohort with limited sample size and statistical power. The survey return rate was $89 \%$ prior to training, $85 \%$ after. While acceptable for this type of research, responses may be systematically different from those who did not complete the surveys, a possible bias. Finally, our evaluation did not capture measures specifically meant to reflect the interprofessional education program. Demonstrating high satisfaction and self-perceived improvements was an important first step. Future work should establish how this influences students' perceptions of working with other professions as well as whether this method of instruction leads to greater learning than the traditional "within profession" approach to training. Next steps should also include examination of potential differences between those for whom this training is/was their sole exposure compared with those with either prior and/or concurrent training (e.g., enrolled in elective course, placement/internship at alcohol/drug use treatment facility, etc.)

\subsection{Strengths}

Our research has important strengths. This is the first report we know of piloting the implementation in an academic setting of SBIRT curriculum on substance use (SU) conditions that included all medical and behavioral students in one cohort under a single, collaborative, interprofessional faculty team. The findings were significantly positive and are generalizable to other settings where training is focused on increasing readiness for collaborative health care using evidence-based interventions in team-based care.

Major findings of this study have implications for future training of a sustainable, high-value workforce. To respond to the significant negative impact of SU conditions, health care professionals need to feel rewarded by working with this patient/client population. Although BH students showed less baseline negative bias toward patients/clients with SU conditions, all trainees found an improved sense of satisfaction with caring for these patients/clients and a reduced sense of helplessness in addressing their problems. These findings, especially amongst PC trainees, may significantly reduce provider burnout (Ratanawongsa et al., 2008) and lead to a 
compelling new hypothesis: interprofessional education may improve both patient/client care and provider experience.

\section{Conclusions}

An IPE program on SBIRT including didactic and interactive practice sessions can improve student knowledge, skills, and attitudes through a practical addition to curricula using self-directed and brief instruction. Future research considerations include comparing intraprofessional education to interprofessional education on the outcomes measured. Key variables to add to this work include knowledge and mutual respect gained during training about other professionals, the impact of the program on future care and outcomes of patients/clients with SUD, and capacity for workforce development for the care of populations with these conditions. Data on provider's change of practice, perceptions of cultural competence with SUD, patient/client perspectives of SBIRT, and their success in changing behavior due to SBIRT would also be valuable. As the SBIRT Advisory Council continues, we plan to continue to measure the benefit of this SBIRT training approach, evaluating the domains of IPE. Programs similar to this one may provide a strategy to sustain the primary care workforce and improve competence in care delivery for patients/clients with SUDs.

\section{Acknowledgments}

The authors wish to thank the other members of the University of Vermont SBIRT Advisory Council:

Carol Buck-Rolland, EdD

Rosemary Dale, EdD

Willow Stein, MSW

Jon van Luling, BA

Gary Widrick, $\mathrm{PhD}$

And also, the leadership of the UVM Clinical Simulation Laboratory:

Cate Nicholas, EdD, MS, PA

Robert Bolyard

And the Director of the Teaching Academy at the University of Vermont:

Kathryn Huggett, PhD, Director, The Teaching Academy at the Larner College of Medicine

\section{Funding/Support}

\section{SAMHSA}

Grantee Federal Identification Number: 1U79TI025395-01

Name of Institution awarded Grant: University of Vermont

Project Name: UVM SBIRT Training Collaborative

The funder provided oversight and technical support during the course of this project but was not involved in the composition of the manuscript.

Note: Dr. Barbara Rouleau passed away unexpectedly in 2017. Although she contributed significantly to the manuscript, she did not approve its final submission.

\section{References}

Agerwala, S. M., \& McCance-Katz, E. F. (2012). Integrating screening, brief intervention, and referral to treatment (SBIRT) into clinical practice settings: a brief review. J Psychoactive Drugs, 44(4), 307-317. https://doi.org/10.1080/02791072.2012.720169

APHA. (2008). Alcohol screening and brief intervention: A guide for public health practitioners. Retrieved from Washington, DC. https://doi.org/10.1300/J465v28n03_03

Babor, T. F., McRee, B. G., Kassebaum, P. A., Grimaldi, P. L., Ahmed, K., \& Bray, J. (2007). Screening, Brief Intervention, and Referral to Treatment (SBIRT): toward a public health approach to the management of substance abuse. Subst Abus, 28(3), 8. https://doi.org/10.1300/J465v28n03_03

Bernstein, E., Bernstein, J., Feldman, J., Fernandez, W., Hagan, M., Mitchell, P., . . Owens, P. (2007). An evidence based alcohol screening, brief intervention and referral to treatment (SBIRT) curriculum for emergency 
department (ED) providers improves skills and utilization. Subst Abus, 28(4), 79-92. https://doi.org/10.1300/J465v28n04_01

Bolesta, S., \& Chmil, J. V. (2014). Interprofessional education among student health professionals using human patient simulation. Am J Pharm Educ, 78(5), 94. https://doi.org/10.5688/ajpe78594

Bray, J. H., Kowalchuk, A., Waters, V., Laufman, L., \& Shilling, E. H. (2012). Baylor SBIRT Medical Residency Training Program: model description and initial evaluation. Subst Abus, 33(3), 231-240. https://doi.org/10.1080/08897077.2011.640160

Cleak, H., \& Williamson, D. (2007). Preparing health science students for interdisciplinary professional practice. $J$ Allied Health, 36(3), 141-149.

Corrigan, J. M. (2005). Crossing the quality chasm: A New Engineering/Health Care Partnership. Washington (DC): National Academies Press (US).

Davoudi, M., \& Rawson, R. A. (2010). Screening, brief intervention, and referral to treatment (SBIRT) initiatives in California: notable trends, challenges, and recommendations. J Psychoactive Drugs, Suppl 6, 239-248. https://doi.org/10.1080/02791072.2010.10400547

Duong, D. K., O'Sullivan, P. S., Satre, D. D., Soskin, P., \& Satterfield, J. (2016). Social Workers as Workplace-Based Instructors of Alcohol and Drug Screening, Brief Intervention, and Referral to Treatment (SBIRT) for Emergency Medicine Residents. Teaching and Learning in Medicine, 28(3), 303-313. https://doi.org/10.1080/10401334.2016.1164049

Gache, P., Michaud, P., Landry, U., Accietto, C., Arfaoui, S., Wenger, O., \& Daeppen, J.-B. (2005). The Alcohol Use Disorders Identification Test (AUDIT) as a Screening Tool for Excessive Drinking in Primary Care: Reliability and Validity of a French Version. Alcoholism: Clinical and Experimental Research, 29(11), 2001-2007. https://doi.org/10.1097/01.alc.0000187034.58955.64

Gilbert, J. H., Yan, J., \& Hoffman, S. J. (2010). A WHO report: framework for action on interprofessional education and collaborative practice. J Allied Health, 39 Suppl 1, 196-197.

Gryczynski, J., Mitchell, S. G., Peterson, T. R., Gonzales, A., Moseley, A., \& Schwartz, R. P. (2011). The relationship between services delivered and substance use outcomes in New Mexico's Screening, Brief Intervention, Referral and Treatment (SBIRT) Initiative. Drug and Alcohol Dependence, 118(2-3), 152-157. doi:https://doi.org/10.1016/j.drugalcdep.2011.03.012

Hackett, A., Rhodes, D, Cox, C. (2015). Attitudes toward Healthcare Teamwork between Osteopathic Medical Students in an Interprofessional or Intraprofessional Clinical Education Program. Health and Interprofessional Practice, 2(3), eP1072. doi:https://doi.org/10.7710/2159-1253.1072

IEC. (2011). Core competencies for interprofessional collaborative practice: Report of an expert panel. Retrieved from Washington, D.C. https://doi.org/10.3389/fpsyg.2013.00863

Lakens, D. (2013). Calculating and reporting effect sizes to facilitate cumulative science: a practical primer for t-tests and ANOVAs. Frontiers in Psychology, 4(863). doi:10.3389/fpsyg.2013.00863

Madras, B. K., Compton, W. M., Avula, D., Stegbauer, T., Stein, J. B., \& Clark, H. W. (2009). Screening, brief interventions, referral to treatment (SBIRT) for illicit drug and alcohol use at multiple healthcare sites: Comparison at intake and 6 months later. Drug and Alcohol Dependence, 99(1-3), 280-295. https://doi.org/10.1016/j.drugalcdep.2008.08.003

Malone, G. P., Vale Arismendez, S., Schneegans Warzinski, S., Amodei, N., Burge, S. K., Wathen, P. I., . . . Williams, J. F. (2015). South Texas Residency Screening, Brief Intervention, and Referral to Treatment (SBIRT) Training: 12-Month Outcomes. Subst Abus, 36(3), 272-280. https://doi.org/10.1080/08897077.2014.988839

Mann, K., Sargeant, J., \& Hill, T. . (2009). Knowledge translation into interprofessional education: What difference does interprofessional education make to practice? . Learning in Health and Social Care, 8(3), 154-164. https://doi.org/10.1111/j.1473-6861.2008.00207.x

Marshall, V. J., McLaurin-Jones, T. L., Kalu, N., Kwagyan, J., Scott, D. M., Cain, G., . . Taylor, R. E. (2012). Screening, brief intervention, and referral to treatment: public health training for primary care. Am J Public Health, 102(8), e30-36. https://doi.org/10.2105/AJPH.2012.300802 
MINT. (2016). MINT: Excellence in Motivational Interviewing. MINT: Excellence in Motivational Interviewing. Retrieved from http://www.motivationalinterviewing.org/

Mitchell, A. M., Riccelli, A., Boucek, L., Puskar, K. R., Hagle, H., \& Lindsay, D. (2018). Effect on Dental Hygiene Students of a Substance Use Simulation Conducted with Nursing Students. Journal of Dental Education, 82(5), 469-474. https://doi.org/10.21815/JDE.018.052

Mitchell, M. A., Broyles, L. M., Pringle, J. L., Kraemer, K. L., Childers, J. W., Buranosky, R. A., \& Gordon, A. J. (2017). Education for the mind and the heart? Changing residents' attitudes about addressing unhealthy alcohol use. Subst Abus, 38(1), 40-42. https://doi.org/10.1080/08897077.2016.1185076

Monteiro, K., Dumenco, L., Collins, S., Bratberg, J., MacDonnell, C., Jacobson, A., . . . George, P. (2017). An interprofessional education workshop to develop health professional student opioid misuse knowledge, attitudes, and skills. Journal of the American Pharmacists Association, 57(2), S113-S117. https://doi.org/10.1016/j.japh.2016.12.069

Neander, L. L., Hanson, B. L., Edwards, A. E., Shercliffe, R., Cattrell, E., Barnett, J. D., . . King, D. K. (2018). Teaching SBIRT through simulation: Educational case studies from nursing, psychology, social work, and medical residency programs. 13, 39-47. https://doi.org/10.1016/j.xjep.2018.08.002

Neft, M. W., Mitchell, A.M., Puskar, K. (2018). Interprofessional education for teaching screening, brief intervention, and referral to treatment (SBIRT) for substance use. Journal of Interprofessional Education and Practice, 10, 12-14. https://doi.org/10.1016/j.xjep.2017.10.004

Neufeld, K. J., Alvanzo, A., King, V. L., Feldman, L., Hsu, J. H., Rastegar, D. A., . . MacKinnon, D. F. (2012). A collaborative approach to teaching medical students how to screen, intervene, and treat substance use disorders. Subst Abus, 33(3), 286-291. https://doi.org/10.1080/08897077.2011.640090

Newhouse, R. P., \& Spring, B. (2010). Interdisciplinary evidence-based practice: moving from silos to synergy. Nurs Outlook, 58(6), 309-317. https://doi.org/10.1016/j.outlook.2010.09.001

Olenick, M., Allen, L. R., \& Smego, R. A., Jr. (2010). Interprofessional education: a concept analysis. Adv Med Educ Pract, $1,75-84$. https://doi.org/10.2147/AMEP.S13207

Pelling, S., Kalen, A., Hammar, M., \& Wahlstrom, O. (2011). Preparation for becoming members of health care teams: findings from a 5-year evaluation of a student interprofessional training ward. J Interprof Care, 25(5), 328-332. https://doi.org/10.3109/13561820.2011.578222

Peterson, J., Brommelsiek, M., Amelung, S.K. (2017). An interprofessional education project to address veteran's healthcare needs. International Journal of Higher Education, 6, 1-16. https://doi.org/10.5430/ijhe.v6n1p1

Pringle, J. L., Kearney, S. M., Rickard-Aasen, S., Campopiano, M. M., \& Gordon, A. J. (2017). A statewide screening, brief intervention, and referral to treatment (SBIRT) curriculum for medical residents: Differential implementation strategies in heterogeneous medical residency programs. Subst Abus, 38(2), 161-167. https://doi.org/10.1080/08897077.2017.1288195

Pringle, J. L., Melczak, M., Johnjulio, W., Campopiano, M., Gordon, A. J., \& Costlow, M. (2012). Pennsylvania SBIRT Medical and Residency Training: developing, implementing, and evaluating an evidenced-based program. Subst Abus, 33(3), 292-297. https://doi.org/10.1080/08897077.2011.640091

Puskar, K., Gotham, H. J., Terhorst, L., Hagle, H., Mitchell, A. M., Braxter, B., . . Burns, H. K. (2013). Effects of Screening, Brief Intervention, and Referral to Treatment (SBIRT) education and training on nursing students' attitudes toward working with patients who use alcohol and drugs. Subst Abus, 34(2), 122-128. https://doi.org/10.1080/08897077.2012.715621

Ratanawongsa, N., Roter, D., Beach, M. C., Laird, S. L., Larson, S. M., Carson, K. A., \& Cooper, L. A. (2008). Physician Burnout and Patient-Physician Communication During Primary Care Encounters. Journal of General Internal Medicine, 23(10), 1581-1588. https://doi.org/10.1007/s11606-008-0702-1

Robben, S., Perry, M., van Nieuwenhuijzen, L., van Achterberg, T., Rikkert, M. O., Schers, H., . . Melis, R. (2012). Impact of interprofessional education on collaboration attitudes, skills, and behavior among primary care professionals. J Contin Educ Health Prof, 32(3), 196-204. https://doi.org/10.1002/chp.21145

SAMHSA. (2017). CSAT GPRA Modernization Act Data Collection Tools. Retrieved from https://www.samhsa.gov/grants/gpra-measurement-tools/csat-gpra 
Satterfield, J. M., O'Sullivan, P., Satre, D. D., Tsoh, J. Y., Batki, S. L., Julian, K., . . Wamsley, M. (2012). Using standardized patients to evaluate screening, brief intervention, and referral to treatment (SBIRT) knowledge and skill acquisition for internal medicine residents. Subst Abus, 33(3), 303-307. https://doi.org/10.1080/08897077.2011.640103

Scott, D. M., McLaurin-Jones, T., Brown, F. D., Newton, R., Marshall, V. J., Kalu, N., . . Taylor, R. E. (2012). Institutional incorporation of screening, brief intervention, and referral to treatment (SBIRT) in residency training: achieving a sustainable curriculum. Subst Abus, 33(3), 308-311. https://doi.org/10.1080/08897077.2011.640135

Seale, J. P., Velasquez, M. M., Johnson, J. A., Shellenberger, S., von Sternberg, K., Dodrill, C., . . Grace, D. (2012). Skills-based residency training in alcohol screening and brief intervention: results from the Georgia-Texas "Improving Brief Intervention" Project. Subst Abus, 33(3), 261-271. https://doi.org/10.1080/08897077.2011.640187

Seif, G., Coker-Bolt, P., Kraft, S., Gonsalves, W., Simpson, K., \& Johnson, E. (2014). The development of clinical reasoning and interprofessional behaviors: service-learning at a student-run free clinic. J Interprof Care, 28(6), 559-564. https://doi.org/10.3109/13561820.2014.921899

Solomon, P., \& Salfi, J. (2011). Evaluation of an interprofessional education communication skills initiative. Educ Health (Abingdon), 24(2), 616.

Stanton, M. R., Atherton, W. L., Toriello, P. J., \& Hodgson, J. L. (2012). Implementation of a "learner-driven" curriculum: an screening, brief intervention, and referral to treatment (SBIRT) interdisciplinary primary care model. Subst Abus, 33(3), 312-315. https://doi.org/10.1080/08897077.2011.640140

Tetrault, J. M., Green, M. L., Martino, S., Thung, S. F., Degutis, L. C., Ryan, S. A., . . D'Onofrio, G. (2012). Developing and implementing a multispecialty graduate medical education curriculum on Screening, Brief Intervention, and Referral to Treatment (SBIRT). Subst Abus, 33(2), 168-181. https://doi.org/10.1080/08897077.2011.640220

Venkat, A., Aldrige, A., Kearney, S. M., Radack, J., Rickard-Assen, S., Grasso, K., . . Pringle, J. (2017). Derivation of a Shortened Research Instrument for Measuring Alcohol and Other Drug (AOD) Attitudes in a Screening, Brief Intervention, and Referral to Treatment (SBIRT) Training Program. Journal of Science, Humanities and Arts, 4(2). https://doi.org/10.17160/josha.4.2.290

Warner, M., Trinidad, J. P., Bastian, B. A., Minino, A. M., \& Hedegaard, H. (2016). Drugs Most Frequently Involved in Drug Overdose Deaths: United States, 2010-2014. Natl Vital Stat Rep, 65(10), 1-15.

WHO. (2015). Alcohol Fact Sheet. Retrieved from http://www.who.int/mediacentre/factsheets/fs349/en/

Yudko, E., Lozhkina, O., \& Fouts, A. A comprehensive review of the psychometric properties of the Drug Abuse Screening Test. Journal of Substance Abuse Treatment, 32(2), 189-198. https://doi.org/10.1016/j.jsat.2006.08.002

\section{Notes}

Note 1. For any information about the survey, please write to the corresponding author by email: Constance.Van-Eeghen@med.uvm.edu.

Note 2. (Bernstein et al., 2007; Bray, Kowalchuk, Waters, Laufman, \& Shilling, 2012; Malone et al., 2015; Marshall et al., 2012; M. A. Mitchell et al., 2017; Neufeld et al., 2012; Pringle, Kearney, Rickard-Aasen, Campopiano, \& Gordon, 2017; Puskar et al., 2013; Satterfield et al., 2012; Scott et al., 2012; Seale et al., 2012; Stanton, Atherton, Toriello, \& Hodgson, 2012; Tetrault et al., 2012)

Note 3. Study data were collected and managed using REDCap electronic data capture tools hosted at the University of Vermont. REDCap (Research Electronic Data Capture) is a secure, web-based application designed to support data capture for research studies. 